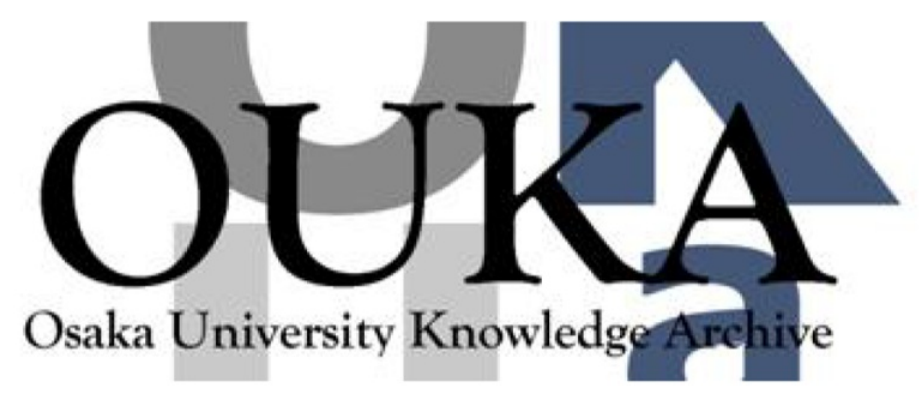

\begin{tabular}{|c|l|}
\hline Title & $\begin{array}{l}\text { Multi-layered flyer accelerated by laser } \\
\text { induced shock waves }\end{array}$ \\
\hline Author(s) & $\begin{array}{l}\text { Tanaka, Kazuo A.; Hara, Motohiko; Ozaki, } \\
\text { Norimasa et al. }\end{array}$ \\
\hline Citation & Physics of Plasmas. 7(2) p. 676-p. 680 \\
\hline Issue Date & $2000-02$ \\
\hline oaire:version VoR \\
\hline URL & https://hdl. handle. net/11094/2887 \\
\hline rights & \\
\hline Note & \\
\hline
\end{tabular}

Osaka University Knowledge Archive : OUKA

https://ir. Library. osaka-u. ac. jp/

Osaka University 


\title{
Multi-layered flyer accelerated by laser induced shock waves
}

\author{
Kazuo A. Tanaka, ${ }^{\text {a) }}$ Motohiko Hara, ${ }^{\text {a) }}$ Norimasa Ozaki, ${ }^{\text {a) }}$ Yasufumi Sasatani, ${ }^{\text {a) }}$ \\ and Sergei I. Anisimov ${ }^{\text {b) }}$ \\ Institute of Laser Engineering, Osaka University, Suita, Osaka 565-0871, Japan \\ Ken-ichi Kondo \\ Institute of Laser Engineering, Osaka University, Suita, Osaka 565-0871, Japan \\ and Materials and Structures Laboratory, Tokyo Institute of Technology, Nagatsutacho 4259, \\ Midori, Yokohama, Kanagawa 227, Japan \\ Motohiro Nakano ${ }^{\text {a) }}$ and Katsunobu Nishihara \\ Institute of Laser Engineering, Osaka University, Suita, Osaka 565-0871, Japan \\ Hisataka Takenaka \\ Institute of Laser Engineering, Osaka University, Suita, Osaka 565-0871, Japan \\ and NTT Advanced Technology Co., Midoricho 3-9-11, Musashino, Tokyo 180, Japan \\ Masatake Yoshida \\ Institute of Laser Engineering, Osaka University, Suita, Osaka 565-0871, Japan \\ and National Institute of Materials and Chemical Research, Higashi 1-1, Tsukuba, Ibaragi 305, Japan \\ Kunioki Mima \\ Institute of Laser Engineering, Osaka University, Suita, Osaka 565-0871, Japan
}

(Received 13 July 1999; accepted 27 September 1999)

Multi-layered flyer (aluminum-polyimide-tantalum) is designed as a high speed flyer making use of shock impedance matching and reverberation techniques. The designed three layered targets have been irradiated using a $20 \mathrm{~J}$ laser beam. Flyer velocities are measured by observing the flyer impact emissions on glass step targets within a $500 \mu \mathrm{m}$ laser focal spot at laser intensities $5 \times 10^{12}-2$ $\times 10^{13} \mathrm{~W} / \mathrm{cm}^{2}$. Thin $(0.5-1.0 \mu \mathrm{m})$ Ta layers of the flyers are accelerated via shock reverberations between the thick polyimide and thin Ta layers for the first time using laser induced shock waves. Their velocities are measured to be more than $13 \mathrm{~km} / \mathrm{s}$ with a good hydrodynamic stability. The obtained velocity is faster than the ones obtained by a conventional flyer method such as a double gas gun. () 2000 American Institute of Physics. [S1070-664X(00)00602-9]

\section{INTRODUCTION}

Shock wave experiments have been a vital tool to establish the EOS of condensed matters up to $100 \mathrm{GPa}$ pressure ranges. ${ }^{1}$ Projectile (flyer) acceleration to high velocities by both explosive acceleration and a two-stage gas gun has been useful for many equation of state (EOS) studies. However, achievable flyer velocities have been practically limited to $5-7 \mathrm{~km} / \mathrm{s}$ for the explosive acceleration and $7-10 \mathrm{~km} / \mathrm{s}$ for the two-stage gas gun. Recently, the use of high-power pulsed lasers has been demonstrated to achieve very high pressures and have been effective to study the EOS of condensed matters. ${ }^{2-5}$ Compressed states have been created mostly by direct or indirect laser illumination methods. One of the shortcomings of these methods is that one has to pay attention to the hot electron or x-ray preheat level in the material before the shock propagation.

Our purpose in this study is to demonstrate a laser driven multi-layered flyer acceleration method. This method could be extended to measure the EOS of matters such as carbon, plastics, and deuterium with a pressure range from 0.1 to 10

\footnotetext{
a) Also at the Faculty of Engineering, Osaka University, Suita, Osaka 565-0871, Japan.

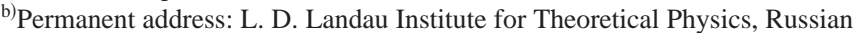
Academy of Sciences, 117940 Moscow, Kosygina St. 2, Russia.
}

$\mathrm{TPa}$, a temperature range from $10^{4}$ to $10^{5} \mathrm{~K}$ free from preheat problems. The preheat problems stem from the laserproduced plasmas, where hot electrons and/or hard $\mathrm{x}$ rays with a long mean free paths could be produced. These hot electrons and hard $\mathrm{x}$ rays could heat up the material to be studied before the shock arrival, since the hot electrons and $\mathrm{x}$ rays propagate much faster than the shock waves. The $\mathrm{x}$ ray preheat could be blocked choosing the proper high $Z$ ( $Z$ : atomic number) materials for the flyer. The hot electron preheat could be suppressed by both using high $Z$ flyer and keeping the distance between the flyer and the impactor larger than a Larmor radius of hot electrons. Normally the hot electrons are ejected out from the laser irradiated target as a beam and could create a azimuthal magnetic field. The hot electrons could be bent with a Larmor radius. The above pressure ranges are predicted in various theoretical models to have effects due to phase transitions such as dissociation and ionization, but a few experiments have been performed with a direct laser irradiation. ${ }^{2,5}$ Measuring EOS under such conditions is essential for inertial confinement fusion (ICF), astrophysics, and the study of new material production. If the scaling of the multi-layered flyer method is established, it could be applied to these fields such as the above as a new scheme. We have designed and performed high-power laser, driven flyer impact experiments with a multi-layered flyer. 


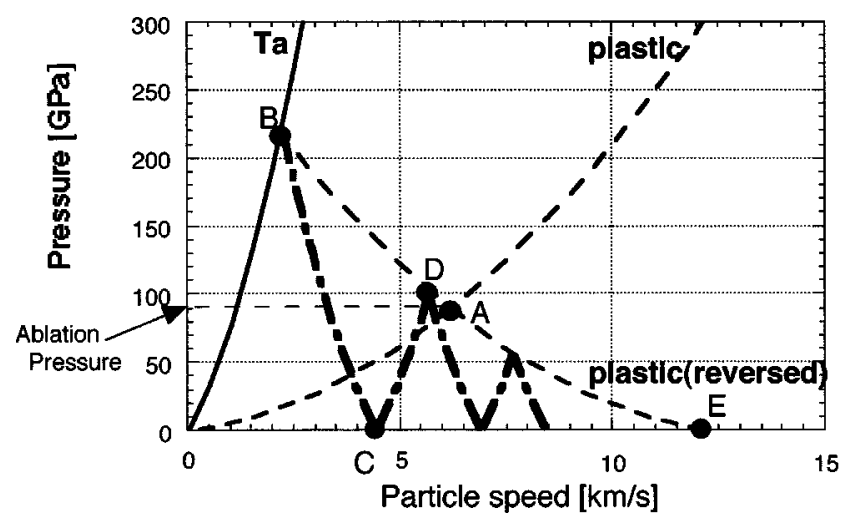

FIG. 1. Schematic of shock impedance matching conditions for Ta and plastic as $\mathrm{P}$ (pressure) $-u_{p}$ (particle velocity) curves. An ablation pressure is applied on the plastic layer at the point A. A shock wave is launched into the plastic and then Ta layer. Obtained pressure is shown as a point B at the boundary of the plastic and Ta layers. Here the plastic curve is reversed to meet the Ta curve at around a given ablation pressure. A rarefaction wave is released in Ta and meets a shock wave in plastic at a point D. At this point $\mathrm{D}$, the Ta layer is accelerated. Similar shock reverberation repeats until the pressure in the plastic becomes zero at a point $\mathrm{E}$. The particle velocity at $\mathrm{E}$ indicates a possible maximum Ta flyer velocity at about $13 \mathrm{~km} / \mathrm{s}$.

Thin Ta flyers are shown to be accelerated up to more than $13 \mathrm{~km} / \mathrm{s}$ making use of the shock reverberation.

\section{EXPERIMENTAL DESIGN}

An advantage of the multi-layered flyers is a potential to achieve a very high velocity free from the preheat. We use a three, layered flyer, consisting of Al-polyimide-Ta layers. A laser beam with a $1053 \mathrm{~nm}$ wavelength irradiates the $\mathrm{Al}$ side of Al-polyimide-Ta multi-layered flyer with an up to $150 \mathrm{GPa}$ ablation pressure at a laser intensity $10^{13} \mathrm{~W} / \mathrm{cm}^{2}$ with a 1 ns pulse width. The Al layer works as a block against laser shine through and/or ablation layer. The shinethrough effect is that the low intensity part of a laser pulse can transmit in a initially transparent medium (polyimide in this case) and may start creating a plasma at the boundary of two materials such as polyimide and Ta (nontransparent for the laser). At the low laser intensities used in our experiment, $\mathrm{Al}$ and polyimide should have more than $50 \%$ absorption efficiency. Thus this thin Al layer was used only for the shine-through block.

Mechanisms of the thin Ta layer acceleration are explained below using Fig. 1. Figure 1 shows that the $P$ (pressure) $-u_{p}$ (particle velocity) graph for Ta and plastic. A plastic curve was used instead of polyimide as a middle layer of the flyer, since no detailed data was available for polyimide. The graph was extrapolated from the existing data region to the pressure region used in this experiment for the explanation of the acceleration mechanisms. These curves were used often to express the shock impedance matching conditions of different materials in a steady state. ${ }^{1}$ A shock wave is launched from the laser ablation layer to a plastic (point A in Fig. 1), then to a Ta layer (point B). Here the plastic curve at around a given ablation pressure was reversed to meet the Ta curve. The shock wave goes quickly through a thin Ta layer. Then an unloading (rarefaction) wave comes back to the plastic. When the unloading wave from the Ta meets the shock wave from the plastic at the plastic-Ta boundary (point D), a shock wave is reflected back into the Ta and accelerates it based on the impedance matching condition. ${ }^{1}$ The Ta layer can be accelerated several times via a shock reverberation as long as the shock wave in the polyimide exists. After the shock reverberation, a final state (point E) could be reached. A possible maximum velocity of Ta is $13 \mathrm{~km} / \mathrm{s}$ or so with an assumption that the plastic layer accelerates the Ta layer ideally until the pressure in the polyimide becomes zero in a steady state (point E). This velocity corresponds to the free surface velocity of polyimide for a given shock pressure. The free surface velocity of Ta could be $5 \mathrm{~km} / \mathrm{s}$ under the ablation pressure of $100 \mathrm{GPa}$. This may be the lower limit of the flyer velocity.

A highest accelerated speed for a thin flyer could be obtained when the polyimide layer should be heated by a strong shock to a plasma temperature and could expand rapidly like an ideal gas. In this limit, an expanding polyimide plasma could accelerate a thin flyer layer as if an explosive accelerates a flyer. If a shock heated polyimide layer is assumed to follow adiabatic expansion, the expansion has a well known relation of $P V^{\gamma}=$ const $(\gamma=2-3)$, where $P$ is the pressure, $V$ is the volume, and $\gamma$ is the parameter characteristic of the expansion. The expansion velocity is expressed as $2 c /(\gamma-1)$, where $c$ is the sound speed. This velocity could be as high as $30 \mathrm{~km} / \mathrm{s}$ for a $\gamma=2$.

As a high speed flyer, a high impedance materials with a high melting temperature should be good. Since Ta has a high melting point $(=3300 \mathrm{~K})$ and high impedance (density $=16.6 \mathrm{~g} / \mathrm{cm}^{3}$ ), we selected Ta as the rear layer of the flyer. Ideally a tailored laser pulse could be a good choice in order not to increase the temperature in the Ta layer, since the tailored pulse is supposed to create series of weak shock with a minimum entropy increase. ${ }^{1,6}$ Even if a flyer acceleration could be estimated in the impedance matching of $P$ (pressure) $-u_{p}$ (particle velocity) curves of the materials, the impedance curves assume a steady state and have no information of time and material thickness. In order to obtain a design of the experiment, such as the timing of both shock and rarefaction waves and to determine each layer thickness, we used a one-dimensional 1D hydrodynamic code. We take into account the ablation pressure profile corresponding to the laser irradiation intensity with a pressure scaling given by Max and Fabbro. ${ }^{7}$ One-dimensional Lagrangian hydrodynamic computations are performed with Wilkins, type artificial viscosity. Laser energy is deposited to a cell which has "critical plasma density,', assuming that the plasma is fully ionized. The critical plasma density is determined so that the single flyer experimental results at roughly identical experimental conditions can be reproduced. The SESAME EOS database $^{8}$ was used for the ablation materials and Ta. Polyimide $\left(1.50 \mathrm{~g} / \mathrm{cm}^{3}\right)$ was substituted by parylene-d $(1.42$ $\left.\mathrm{g} / \mathrm{cm}^{3}\right)$. A linear $U_{s}-u_{p}$ relationship and Mie-Gruneisen EOS was used for the flyer material (Ta). We performed 1D simulations for Al-parylene-Ta 3-layered flyer (thickness of each layer: Al 0.2 -parylene 20 - Ta $(0.5-8 \mu \mathrm{m})$ to have guidelines for the target designs. Results from the 1D simulation are shown in Fig. 2, where a Ta flyer speed $(\mathrm{km} / \mathrm{s})$ is on the vertical axis and distance $(\mu \mathrm{m})$ is on the horizontal. 


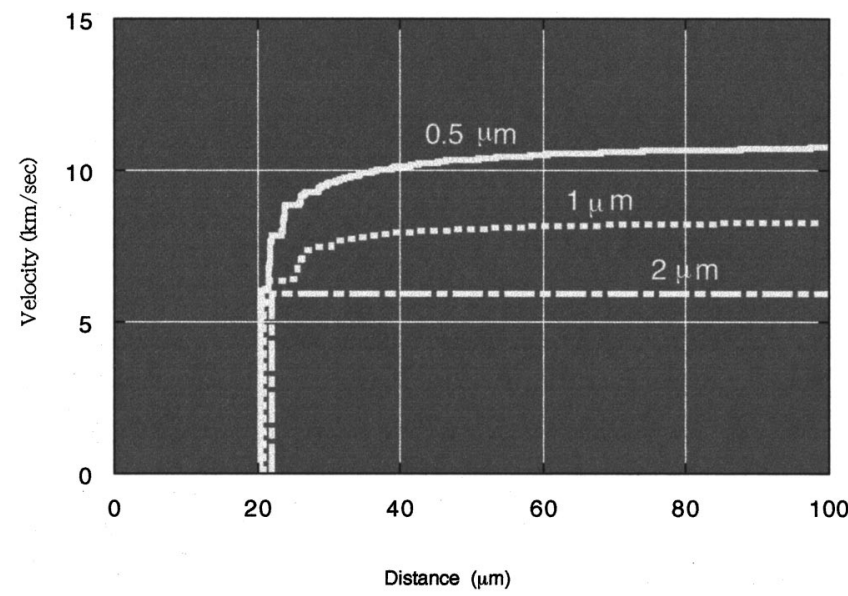

FIG. 2. One dimensional simulation results for multi-layered flyer acceleration. Al $(0.2 \mu \mathrm{m})$, plastic $(20 \mu \mathrm{m}), \mathrm{Ta}(0.5,1.0,2.0 \mu \mathrm{m})$ are used as a multi-layered flyer. On the Al side, an ablation pressure of $150 \mathrm{GPa}$ is applied with a $1 \mathrm{~ns}$ Gaussian temporal pulse. For the case of 0.5 and $1.0 \mu \mathrm{m}$ Ta cases, a clear step-like acceleration is shown indicating that the Ta layers are accelerated with a shock reverberation mentioned earlier. The Ta layers reach their maximum velocities after several ns. The cases of Ta layers thicker than $2 \mu \mathrm{m}$ show almost curves similar to the $2 \mu \mathrm{m}$. These thick Ta flyers did not show step-like acceleration, but a particle velocity after the release point (point $\mathrm{C}$ ) of the first rarefaction wave in Fig. 1.

Ablation pressure is $53 \mathrm{Gpa}$, which corresponds to a laser intensity $10^{13} \mathrm{~W} / \mathrm{cm}^{2}$ with a $1 \mathrm{~ns}$ temporally Gaussian pulse. One-dimensional simulation results were used to find the acceleration effects of the Ta flyer varying the thickness of each layer. This pressure is applied on the $\mathrm{Al} /$ parylene layer and then the shock reverberation in the Ta layer and subsequent acceleration was looked for. As explained earlier, the pressure reverberation in the thin flyer layer results in a stepwise acceleration to high velocities. Each step in the acceleration history corresponds to the timing when an unloading wave from Ta meets a shock wave from the parylene layer. A highest speed is obtained for $\mathrm{Al}(0.2 \mu \mathrm{m})-\mathrm{CH}(20$ $\mu \mathrm{m})-\mathrm{Ta}(0.5 \mu \mathrm{m})$, while the $\mathrm{Al}(0.2 \mu \mathrm{m})-\mathrm{CH}(20 \mu \mathrm{m})-\mathrm{Ta}$ $(1 \mu \mathrm{m})$ case indicates also an acceleration effect. The pressure reverberation does not occur and the Ta layer velocities are free surface velocities $(6 \mathrm{~km} / \mathrm{s})$ without any acceleration when Ta layers are thicker than $2 \mu \mathrm{m}$. Thus the acceleration effects depend on the Ta layer thickness. Since we have not measured the Ta layer temperature in this experiment, it was difficult to discuss further details of the Ta phase state. Most probably the Ta flyer could be in a liquid or partially plasma state at a time of acceleration or shock reverberation. A more detailed calculation of the state of Ta layer was performed by Oparin ${ }^{9}$ using a similar 1D hydrocode with multiphase EOS data taken from Ref. 10. According to these simulation results, the $1 \mu \mathrm{m}$ Ta layer was indicated to be in a two-phase state (30\% solid and $70 \%$ liquid) at a time instant $40 \mathrm{~ns}$ after the ablation pressure application. The detailed study of the Ta state is beyond the scope of this study and should be measured thoroughly in the near future.

\section{EXPERIMENT}

Our experiment was conducted using the GMII (the second generation of glass laser module) laser system at the Institute of Laser Engineering, Osaka University. The layout

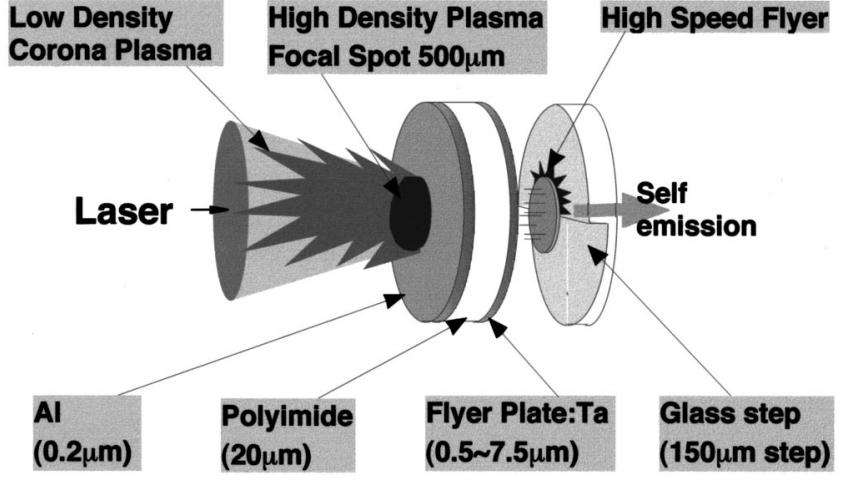

FIG. 3. Schematic view of a multi-layered flyer (aluminum-plastictantalum) for laser intensities $0.5-20 \times 10^{13} \mathrm{~W} / \mathrm{cm}^{2}$, the laser wavelength $1053 \mathrm{~nm}$, and the pulse width $1 \mathrm{~ns}$ (FWHM). Ta thickness varies from 0.5 to $7.5 \mu \mathrm{m}$. Polyimide thickness is $20 \mu \mathrm{m}$ and Al thickness is $0.2 \mu \mathrm{m}$. Flyer velocities are measured from the self-light-emissions at the impact surface of glass steps separated typically by $150 \mu \mathrm{m}$.

of the experiment is shown in Fig. 3. A plane three layer flyer target is driven by a single pulse high-power laser beam with a $1053 \mathrm{~nm}$ wavelength, a $1 \mathrm{~ns}$ pulse width (full width at the half maximum). The driving laser is focused on a flyer surface with a laser energy up to $20 \mathrm{~J}$, with a $500 \mu \mathrm{m}$ spot diam. The ablated surface can generate a shock pressure up to $150 \mathrm{GPa}$ and a shock wave propagates into a multi-layered flyer. The Ta layer of the flyer is accelerated by the mechanisms mentioned earlier and is impacted on glass steps. The first glass step is placed at $100 \mu \mathrm{m}$ from the flyer rear surface. Flyer velocities are measured from the self-lightemissions at the impact surface of glass steps separated typically by $150 \mu \mathrm{m}$. Images of impact signals are optically relayed to the slit of a temporal streak camera by a lens. A time resolution is $0.4 \mathrm{~ns}$ and a spatial resolution is $4 \mu \mathrm{m}$ at a $100 \mathrm{~ns} / 15 \mathrm{~mm}$ sweep mode. Light emissions signals are recorded as digital charge coupled device (CCD) data (492 $\times 512$ pixels).

The $\mathrm{Ta}$ and $\mathrm{Al}$ layers were deposited onto polyimide films using a rf magnetron sputtering deposition system. 0.2 $\mu \mathrm{m}$ of $\mathrm{Al}$ is coated on the polyimide layer. The laser light absorption and subsequent ablation do not make much difference of $\mathrm{Al}$ and polyimide at our relatively low laser intensity regimes, though the thin Al layer should be ablated away quickly at an early stage of the laser pulse. The thickness of Ta layers are chosen to be $0.5,1.0,2.0,4.0$, and $7.5 \mu \mathrm{m}$ to find the acceleration effect predicted in the $1 \mathrm{D}$ simulation. $\mathrm{Al}$ and polyimide thickness are kept same for all targets to be 0.2 and $20 \mu \mathrm{m}$, respectively. The accuracy of coated thickness is $\pm 0.01 \mu \mathrm{m}$.

Typical flyer impact signals for $1 \mu \mathrm{m}$ Ta are shown in Fig. 4. Al $(0.2 \mu \mathrm{m})$-polyimide $(20 \mu \mathrm{m})-\mathrm{Ta}(1 \mu \mathrm{m})$ flyer was irradiated at a laser intensity $1.0 \times 10^{13} \mathrm{~W} / \mathrm{cm}^{2}$ in Fig. 4(a). Two signals of light-emission at the impact surface have a $17.2 \mathrm{~ns}$ time interval and the height of the glass step is $149 \mu \mathrm{m}$, resulting in the flyer velocity of $7.9 \mathrm{~km} / \mathrm{s}$. As shown in Fig. 2 of the simulation results, thin Ta layers reach their maximum velocities after several ns after the laser irradiation and fly with a constant velocity. Thus we measure the constant flyer velocities at the glass step. Al $(0.2 \mu \mathrm{m})-$ polyimide $(20 \mu \mathrm{m})-\mathrm{Ta}(1 \mu \mathrm{m})$ was irradiated at 1.5 


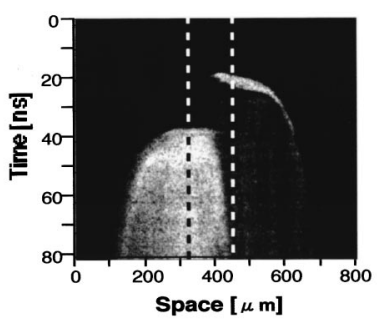

(a)

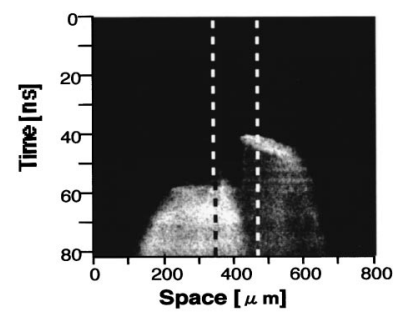

(c)

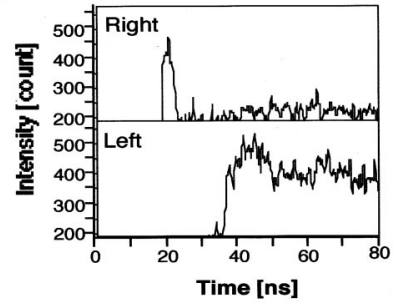

(b)

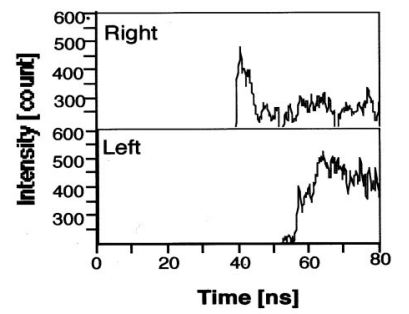

(d)
FIG. 4. (a) Streak image of signals of light-emission at the impact surface with a three-layer flyer of Al $0.2 \mu \mathrm{m}$-polyimide $20 \mu \mathrm{m}$-Ta $1 \mu \mathrm{m}$ irradiated at a laser intensity $1.0 \times 10^{13} \mathrm{~W} / \mathrm{cm}^{2}$. (b) Intensity scans of (a). The white dotted lines show the scanned positions. The light signals at the glass step have a $18.8 \mathrm{~ns}$ time interval and the height of the glass step is $149 \mu \mathrm{m}$, resulting in the flyer velocity of $7.9 \mathrm{~km} / \mathrm{s}$. (c) Streak image of signals of light-emission at the impact surface Al $0.2 \mu \mathrm{m}$-polyimide $20 \mu \mathrm{m}$-Ta $1 \mu \mathrm{m}$ irradiated at $1.5 \times 10^{13} \mathrm{~W} / \mathrm{cm}^{2}$. (d) Intensity scans of (c). The light signals at the glass step have a $17.7 \mathrm{~ns}$ time interval, resulting in the flyer velocity of $9.9 \mathrm{~km} / \mathrm{s}$.

$\times 10^{13} \mathrm{~W} / \mathrm{cm}^{2}$ as shown in Fig. 4(c), where the flyer achieved $9.9 \mathrm{~km} / \mathrm{s}$. The flyer velocities are determined at $100 \mu \mathrm{m}$ from the step boundary to both right and left sides, where the step heights are measured accurately before the laser irradiation. Time steps and step heights have $1 \%$ errors, which may end up with 5\% uncertainties in velocities including reading and streak camera sweep speed errors. In the experimental results, the impact emissions are slightly inclined and curved. These inclinations are reproducible from shot to shot and are due to the focused laser intensity profile.

As clearly seen in both experimental figures of Figs. 4(b) and $4(\mathrm{~d})$, the upper temporal intensity profile corresponding to the right side impact emission in Figs. 4(a) and 4(c) shows a rather sharp spiky emission. The lower intensity profile corresponding to the left side impact emission shows a sharply rising and slowly decaying emission. We expect an emission such as the lower intensity profile for an impact emission. We attribute the spiky emissions seen on the glued first glass steps to a rarefaction wave from the side edge of the step. The rarefaction may come in from the glass step after the flyer impacts on the first step. Since a different kind of glass was attached to a glass substrate, the emission on the right side could be also affected.

Figure 5 shows a dependence of the flyer velocity on the laser intensity $\mathrm{Ta}$ thickness varying from 0.5 to $7.5 \mu \mathrm{m}$. The experimental data are indicated as points, while the solid line shows the estimated Ta free surface velocities, and the dotted line shows the possible maximum velocities by impedance matching method from the simulation results. The experimental data appear to have two groups. In the case of Ta 0.5 $\mu \mathrm{m}(-)$ thickness, observed flyer velocities are faster than the others and became close to the possible maximum veloci-

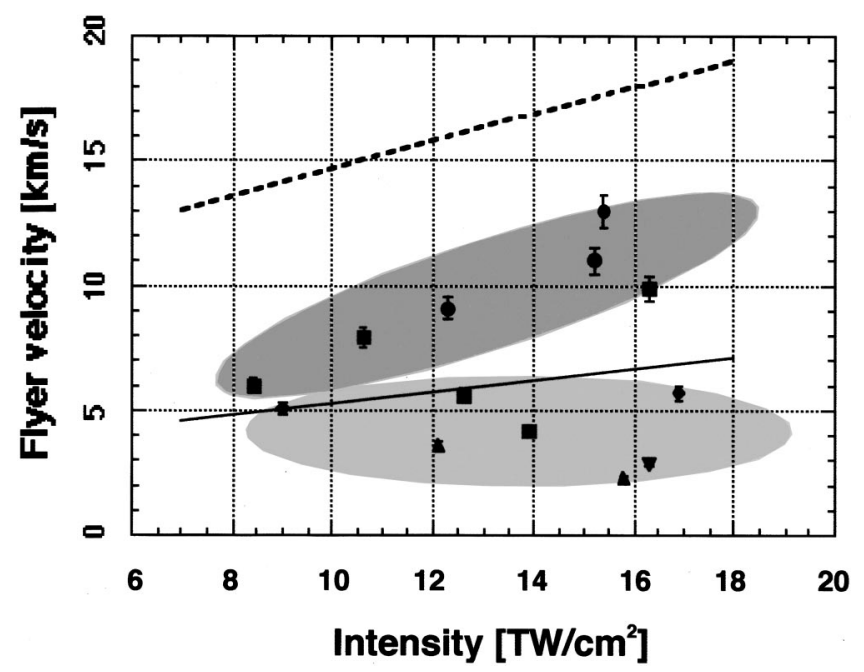

FIG. 5. Observed dependence of flyer velocity on Ta thickness varying from 0.5 to $7.5 \mu \mathrm{m}$. - marks are $\mathrm{Al} 0.2 \mu \mathrm{m}$-polyimide $20 \mu \mathrm{m}$-Ta $0.5 \mu \mathrm{m}$ flyers. Similarly, $\square$ for Al 0.2-polyimide 20-Ta 1; $\bullet$ for Al 0.2-polyimide 20-Ta 2; $\boldsymbol{\Delta}$ for Al 0.2 -polyimide 20 -Ta $4 ; \boldsymbol{\nabla}$ for Al 0.2-polyimide 20-Ta 7.5. The solid line shows the estimated Ta free surface velocities, and the dotted line shows the possible maximum velocities from the simulation. There are two groups of data: one close to the possible maximum and the other close to the free surface velocities, indicating that the Ta flyers thinner than $2 \mu \mathrm{m}$ are effectively accelerated.

ties, consistent with our simulation results. Ta $1 \mu \mathrm{m}(\boldsymbol{\square})$ shows also the increase of the flyer velocities with the laser intensity. The faster flyer speed group shows a gradual increase of the Ta flyer speed from $6 \mathrm{~km} / \mathrm{s}$ up to almost 13 $\mathrm{km} / \mathrm{s}$. Ta $2 \mu \mathrm{m}(\diamond), 4 \mu \mathrm{m}(\boldsymbol{\Delta})$, and $7.5 \mu \mathrm{m}(\boldsymbol{\nabla})$ data sets stay near the solid line. For the thicker flyers, the velocities did not increase as the laser intensity increased and stayed within 2 to $6 \mathrm{~km} / \mathrm{s}$ range. They are basically free surface velocities, showing no sign of the acceleration. There are three data points of Ta $1 \mu \mathrm{m}(\boldsymbol{\square})$ in the higher speed group and two in the slower speed group, indicating that some 1 $\mu \mathrm{m}$ (ם) Ta flyers were actually accelerated and some are not. We will discuss this point later. The flyer speed dependence on the laser intensity is quite similar to the simulated ones for both velocity groups, though there is a $25 \%$ difference in the velocities for the case of the accelerated group. The difference between the observed flyer and the simulated speeds should not be taken seriously, since the simulation was preformed to obtain a design guide line for the experiment. EOS data used for the simulation is actually parylene, not polyimide.

\section{FLYER STABILITY ANALYSIS}

Thin flyers such as the ones here could be subject to hydrodynamic instabilities. We particularly focused on the Richtmyer-Meshkov instability ( $\mathrm{R}-\mathrm{M}$ instability) occurring in the polyimide-Ta interface of a multi-layered flyer. ${ }^{11}$ Here a liquid or partial plasma states is assumed for the polyimide and Ta layers. In R-M instability, the equation of motion about the amplitude of initial perturbation $\xi$ is given by

$$
\frac{d^{2}}{d t^{2}} \xi=\alpha_{A} \Delta U \delta(t) k \xi_{0},
$$


where $k, \Delta U$, and $\delta(t)$ are the wave number, the $\mathrm{Al}-\mathrm{CH}$ interface velocity, and the delta function. $\alpha_{A}$ is a constant called the Atwood number:

$$
\alpha_{A}=\frac{\rho_{2}-\rho_{1}}{\rho_{2}+\rho_{1}} \approx 0.88,
$$

where $\rho_{1}\left(=1 \mathrm{~g} / \mathrm{cm}^{3}\right)$ and $\rho_{2}\left(=16.6 \mathrm{~g} / \mathrm{cm}^{3}\right)$ are the densities of $\mathrm{CH}$ and Ta. The amplitude growth from the initial perturbation can be estimated by integrating Eq. (1) between the shock duration at the polyimide-Ta interface. In our experiment, the amplitude of initial perturbation $\xi_{0}$ is about $10 \AA$, which has been measured by a displacement interferometer on the polyimide-Ta interface. The shock duration is $1 \mathrm{~ns}$, the same as the laser pulse. For the ablation pressure 100 GPa with $20 \mathrm{~J}$ of the laser energy, $\Delta U$ is $4.95(\mathrm{~km} / \mathrm{s})$. In order that the amplitude growth after $1 \mathrm{~ns}$ from the shock front arriving the interface is smaller than $\sim 1 / 10$ of the Ta layer thickness $\mathrm{Ta}$, the wave number $k$ must satisfy the inequality

$$
4.36 \times 10^{-15} k<\frac{\mathrm{Ta}}{10}-10^{-9} .
$$

From Eq. (3), we obtain a condition of the wavelength of an initial perturbation,

$$
\lambda>0.28 \mu \mathrm{m} \text {. }
$$

This shows that, if the wavelength $\lambda$ is larger than $0.28 \mu \mathrm{m}$, the perturbation growth by hydrodynamic instability is sufficiently small and can be neglected. Our focal spot pattern of a $500 \mu \mathrm{m}$ diam had nonuniformity of the order of $10 \mu \mathrm{m}$ scale or larger. Then this laser profile nonuniformity seen within a typical laser focal satisfies the relation of Eq. (4) and may not be a problem for the shock induced instability. The perturbed shock wave fronts created at the ablation surface ripples and their perturbed propagation can be well damped along the propagation. ${ }^{12}$ This damping factor adds to the above estimation and we considered that the Ta thin layers could fly with a good hydrodynamic stability.

Experimental results show a clear acceleration for the Ta flyer thinner than $1 \mu \mathrm{m}$ and free surface velocity for these thicker than $1 \mu \mathrm{m}$. Several mechanisms could be responsible for the observed threshold of the acceleration. One possible explanation is due to spallation: a failure process that occurs near the rear free surface due to the crossing of two releasing waves. One is the rarefaction wave after shock reaches the Ta free surface and the other rarefaction wave may come in following a shock wave from the ablation surface. ${ }^{13-15}$ When these two rarefaction waves encounter, the material is pulled apart and is led to the spallation. After the spallation occurs a flyer is separated into two; one is a scab, which expands with a Ta free surface velocity,' another is simply not accelerated. The phenomenon occurs inside the Ta layer when Ta is thicker than $2 \mu \mathrm{m}$ according to our simple estimation. The spallation phenomenon was observed also in our 1D simulations. Our data in Fig. 5 indicates that the Ta $1 \mu \mathrm{m}$ case may have experience with the spallation, though no spallation was observed in the simulation. There could be a threshold in Ta thickness in the experiment where the spallation may occur. These arguments may indicate that the acceleration threshold is at $1 \mu \mathrm{m}$ and the acceleration effect is only possible for flyers thinner than this value.

\section{CONCLUSION}

We have performed experiments of multi-layered flyer acceleration by laser induced shock waves. Ta thin layers are accelerated as a plane foil at $2-13 \mathrm{~km} / \mathrm{s}$ velocity range with a good hydrodynamic stability. In the case of Ta $1 \mu \mathrm{m}$ and $0.5 \mu \mathrm{m}$, a clear acceleration effect was shown. The acceleration velocities vary with each thickness of $\mathrm{Al}-\mathrm{CH}-\mathrm{Ta}$ layers. The shock reverberations in the Ta layer and the subsequent shock and rarefaction waves encountered at the polyimide-Ta boundary achieve these accelerations. The present laser-driven flyer technique can be applied to EOS experiments possibly free from hot electron and $\mathrm{x}$-ray preheat using much larger laser systems to obtain accurate EOS data of various materials.

\section{ACKNOWLEDGMENTS}

We acknowledge many fruitful discussions with Dr. L. Da Silva, Dr. A. Ng, Dr. R. Cauble, and Dr. P. Cellier at the Lawrence Livermore National Laboratory. The experiment was only possible with the engineering support by the laser operation group, especially Mr. K. Suzuki and K. Sawai. We thank Dr. Y. Kitagawa and Dr. R. Kodama for their interest in this research, and Dr. A. Oparin for presenting his simulation results. S.A. is grateful to the Institute of Laser Engineering, Osaka University for offering the guest professor position. This work has been supported by CREST (Core Research for Evolutional Science and Technology) program of Japan Science and Technology Corporation (JST).

${ }^{1}$ G. E. Euvall and G. R. Rowles, High Pressure Physics and Chemistry, edited by R. S. Brandly (Academic, New York, 1963), Vol. 2, p. 209.

${ }^{2}$ L. B. Da Silva, P. Colliers, G. W. Collins et al., Phys. Rev. Lett. 78, 483 (1997).

${ }^{3}$ S. Fu, Y. Gu, J. Wu, and S. Wang, Phys. Plasmas 2, 3461 (1995).

${ }^{4}$ R. Cauble, D. W. Phillion, T. J. Hoover, N. C. Holmes, J. D. Kikenny, and R. W. Lee, Phys. Rev. Lett. 70, 2102 (1993).

${ }^{5}$ M. Koenig, B. Faral, J. M. Boundenne, D. Batani, A. Benuzzi, S. Bossi, C. Remond, J. P. Perrine, M. Temporal, and S. Atzeni, Phys. Rev. Lett. 74, 2260 (1995).

${ }^{6}$ A. Djaoui, Phys. Plasmas 3, 4677 (1996).

${ }^{7}$ C. E. Max, Les Houches 1980 Session XXXIV, Laser Plasma Interactions, edited by R. Balian and J. C. Adam (North-Holland, Amsterdam, 1982); R. Fabbro, B. Faral, J. Virmont, F. Cottet, and J. P. Romain, Phys. Fluids B 1, 644 (1989).

${ }^{8}$ See National Technical Information Service Document No. DE85011902 (Los Alamos National Laboratory report LA-10160-MS, by K. Holion, 1984). Copies may be ordered from the National Technical Information Service, Springfield, Virginia 22161.

${ }^{9}$ A. M. Oparin (private communication, 1997).

${ }^{10}$ A. V. Bushman, I. V. Lomonosov, and V. E. Fortov, Equations of State of Metals at High Energy Densities, Institute of Chemical Physics (Russian Academy of Sciences, Chernogolovka, 1992).

${ }^{11}$ K. O. Mikaelian, Phys. Rev. A 42, 3400 (1990).

${ }^{12}$ R. Ishizaki and K. Nishihara, Phys. Rev. Lett. 78, 1920 (1997).

${ }^{13}$ R. G. McQeen, S. P. Marsh, J. W. Taylor, J. N. Fritz, and W. J. Carter, in High Velocity Impact Phenomena (Academic, London, 1970).

${ }^{14}$ L. Davidson and R. A. Graham, Phys. Rep. 55, 255 (1979).

${ }^{15}$ M. Boustie and F. Cottet, J. Appl. Phys. 69, 7533 (1991). 